\title{
From DAMA/Nal to DAMA/LIBRA at LNGS
}

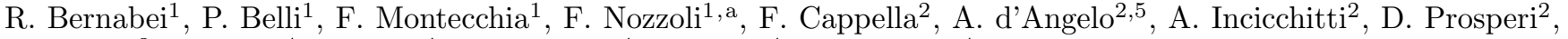 \\ R. Cerulli ${ }^{3}$, C.J. Dai ${ }^{4}$, H.L. He ${ }^{4}$, H.H. Kuang ${ }^{4}$, J.M. $\mathrm{Ma}^{4}$, and Z.P. Ye ${ }^{4}$ \\ 1 Dipartimento di Fisica, Università di Roma "Tor Vergata" and INFN, Sezione di Roma II, I-00133, Roma, Italy \\ 2 Dipartimento di Fisica, Università di Roma "La Sapienza" and INFN, Sezione di Roma, I-00185, Roma, Italy \\ 3 INFN - Laboratori Nazionali del Gran Sasso, I-67010 Assergi (AQ), Italy \\ 4 IHEP, Chinese Academy, P.O. Box 918/3, Beijing 100039, PRC \\ 5 Scuola di Specializzazione in Fisica Sanitaria, Università di Roma "Tor Vergata", I-00133, Roma, Italy
}

Received: 20 June 2005 /

Published online: 22 February 2006 - C Società Italiana di Fisica / Springer-Verlag 2006

\begin{abstract}
DAMA is an observatory for rare processes based on the development and use of various kinds of radiopure scintillators; it is operative deep underground at the Gran Sasso National Laboratory of the INFN. Several low background setups have been realized and many rare processes have been investigated. In particular, the DAMA/NaI setup ( $\simeq 100 \mathrm{~kg}$ highly radiopure $\mathrm{NaI}(\mathrm{Tl}))$ has effectively investigated the model-independent annual modulation signature; the data of seven annual cycles (total exposure of $107731 \mathrm{~kg} \times \mathrm{d}$ ) have offered a $6.3 \sigma$ C.L. model-independent evidence for the presence of a Dark Matter particle component in the galactic halo. Some of the many possible corollary model-dependent quests for the candidate particle have also been investigated. At present, the second-generation DAMA/LIBRA setup $(\simeq 250 \mathrm{~kg}$ highly radiopure $\mathrm{NaI}(\mathrm{Tl}))$ is in operation deep underground. This paper summarizes the main aspects.
\end{abstract}

PACS. 95.35.+d Dark matter (stellar, interstellar, galactic, and cosmological) - 29.40.Mc Scintillation detectors

\section{Introduction}

DAMA is an observatory for rare processes based on the development and use of various kinds of radiopure scintillators. Several low background setups have been realized; the main ones are: i) DAMA/NaI $(\simeq 100 \mathrm{~kg}$ of highly radiopure $\mathrm{NaI}(\mathrm{Tl})$ ), which took data underground over seven annual cycles and was put out of operation in July $2002[1,2,3,4,5,6,7,8,9,10,11,12,13,14]$; ii) DAMA/LXe ( $\simeq 6.5 \mathrm{~kg}$ liquid xenon) $[15]$; iii) DAMA/R\&D, which is devoted to tests on prototypes and small-scale experiments [16]; iv) the new second-generation DAMA/LIBRA setup $(\simeq 250 \mathrm{~kg}$ highly radiopure $\mathrm{NaI}(\mathrm{Tl}))$ in operation since March 2003. Moreover, in the framework of devoted $\mathrm{R} \& \mathrm{D}$ for radiopure detectors and photomultipliers, sample measurements are regularly carried out by means of the low background DAMA/Ge detector, installed deep underground for more than 10 years and, in some cases, by means of Ispra facilities.

In the following, we will focus our attention only on the DAMA/NaI results, mainly recalling the investigation of the annual modulation signature due to a Dark Matter (DM) particle component in the galactic halo. At present, apart DAMA/LIBRA, no other experiment is sensitive,

\footnotetext{
a e-mail: Francesco.Nozzoli@roma2.infn.it
}

for mass and stability, to such a signature. The modelindependent annual modulation signature (originally suggested in [17]) is very distinctive since it requires the simultaneous satisfaction of all the following requirements: the rate must contain a component modulated according to a cosine function (1) with one year period, $T$ (2) and a phase, $t_{0}$, that peaks around $\simeq 2$ nd June (3); this modulation must only be found in a well-defined lowenergy range, where the Dark Matter particle can induce signals (4); it must apply to those events in which just one detector of many actually "fires" (single-hit events), since the DM particle multi-scattering probability is negligible (5); the modulation amplitude in the region of maximal sensitivity is expected to be $\sim 7 \%$ for usually adopted dark halo distributions (6), but it can be larger in case of some possible scenarios such as, e.g., those in refs. [18, 19]. To mimic such a signature spurious effects or side reactions should be able both to account for the whole observed modulation amplitude and to contemporaneously satisfy all the requirements; none has been found $[2,3]$.

Some of the main topics related to this argument on the DAMA/NaI results are shortly summarized in the following; for a detailed discussion see refs. [2,3] and references therein. For a description of the setup and its performances see refs. $[1,2,3,10]$. 

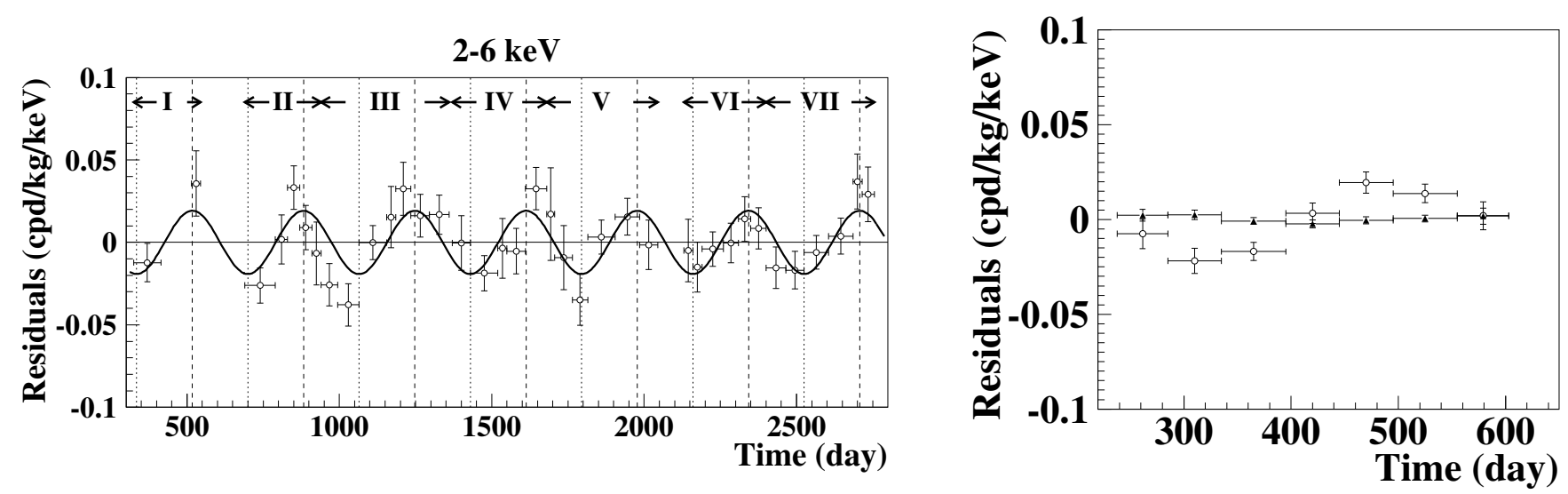

Fig. 1. On the left: experimental residual rate for single-hit events in the cumulative (2-6) keV energy interval as a function of the time over 7 annual cycles (total exposure $107731 \mathrm{~kg} \times \mathrm{d}$ ); end of data taking, July 2002. The experimental points show the errors as vertical bars and the associated time bin width as horizontal bars. The superimposed curve represents the cosinusoidal function behaviour expected for a Dark Matter particle signal with a period equal to 1 y and phase exactly on 2nd June; the modulation amplitude has been obtained by best fit. On the right: experimental residual rates over seven annual cycles for single-hit events (open circles) — class of events to which Dark Matter particle events belong- and over the last two annual cycles for multiple-hits events (filled triangles) — class of events to which Dark Matter particle events do not belong-in the (2-6) keV cumulative energy interval. They have been obtained by considering for each class of events the data as collected in a single annual cycle and using in both cases the same identical hardware and the same identical software procedures. The initial time is taken on August 7th [2,3].

\section{The model-independent result of DAMA/Nal}

A model-independent approach on the data collected by DAMA/NaI over seven annual cycles offers an immediate evidence of the presence of an annual modulation of the measured rate of the single-hit events in the lowestenergy region. In particular, in fig. 1, left, the time behaviour of the residual rate of the single-hit events in the cumulative (2-6) keV energy interval is reported. The data favour the presence of a modulated cosine-like behaviour $\left(A \cdot \cos \omega\left(t-t_{0}\right)\right)$ at $6.3 \sigma$ C.L. and their fit for this cumulative energy interval offers modulation amplitude equal to $(0.0200 \pm 0.0032) \mathrm{cpd} / \mathrm{kg} / \mathrm{keV}, t_{0}=(140 \pm 22) \mathrm{d}$ and $T=\frac{2 \pi}{\omega}=(1.00 \pm 0.01) \mathrm{y}$, all parameters kept free in the fit. The period and phase agree with those expected in the case of an effect induced by Dark Matter particles in the galactic halo $\left(T=1 \mathrm{y}\right.$ and $t_{0}$ roughly at $\simeq 152.5$ th day of the year). The $\chi^{2}$ test on the $(2-6) \mathrm{keV}$ residual rate disfavours the hypothesis of unmodulated behaviour giving a probability of $7 \cdot 10^{-4}\left(\chi^{2} /\right.$ d.o.f. $\left.=71 / 37\right)$. The same data have also been investigated by a Fourier analysis: a clear peak corresponding to a period of $\simeq 1 \mathrm{y}$ is present $[2,3]$. Modulation is not observed above $6 \mathrm{keV}^{1}$. Finally, a suitable statistical analysis has shown that the modulation amplitudes are statistically well distributed in all the crystals, in all the data taking periods and considered energy bins.

\footnotetext{
1 We recall that DAMA/NaI took data up to the MeV energy region despite the optimization was done for the $\mathrm{keV}$ energy range.
}

As a further relevant investigation, the multiple-hits events collected during the DAMA/NaI-6 and 7 running periods (when each detector was equipped with its own Transient Digitizer with a dedicated renewed electronics) have been studied and analysed by using the same identical hardware and the same identical software procedures as for the case of the single-hit events (see fig. 1, right). The multiple-hits event class - on the contrary of the single-hit one- does not include events induced by Dark Matter particles since the probability that a Dark Matter particle interacts in more than one detector is negligible. The obtained modulation amplitudes are: $A=(0.0195 \pm 0.0031) \mathrm{cpd} / \mathrm{kg} / \mathrm{keV}$ and $A=-(3.9 \pm 7.9)$. $10^{-4} \mathrm{cpd} / \mathrm{kg} / \mathrm{keV}$ for single-hit and multiple-hits residual rates, respectively. Thus, evidence of annual modulation is present in the single-hit residuals (event class to which the Dark Matter particle induced events belong), while it is absent in the multiple-hits residual rate (event class to which only background events belong). Since the same identical hardware and the same identical software procedures have been used for the two classes of events, the obtained result offers an additional strong support for the presence of Dark Matter particles in the galactic halo further excluding any side effect either from hardware or from software procedures or from background.

Moreover, a careful investigation of all the known possible sources of systematics and side reactions has been regularly carried out and published at time of each data release and quantitative discussions can be found in refs. [2, $3,10]$. No systematic effect or side reaction able to account for the observed modulation amplitude and to satisfy all the requirements of the signature has been found. Thus, very cautious upper limits (90\% C.L.) on the possible con- 
Table 1. Summary of the results obtained by investigating the possible sources of systematics or of side reactions $[2,3]$. No systematics or side reaction has been found able to give a modulation amplitude different from zero; thus very cautious upper limits (90\% C.L.) have been calculated and are shown here in terms of the measured model-independent modulation amplitude, $S_{m}^{o b s}$. As can be seen, they cannot account for the measured modulation amplitude and, in addition, cannot satisfy the peculiar requirements of the signature.

\begin{tabular}{|c|c|}
\hline Source & $\begin{array}{l}\text { Cautious upper limit } \\
(90 \% \text { C.L. })\end{array}$ \\
\hline Radon & $<0.2 \% S_{m}^{o b s}$ \\
\hline Temperature & $<0.5 \% S_{m}^{o b s}$ \\
\hline Noise & $<1 \% S_{m}^{o b s}$ \\
\hline Energy scale & $<1 \% S_{m}^{o b s}$ \\
\hline Efficiencies & $<1 \% S_{m}^{o b s}$ \\
\hline Background & $<0.5 \% S_{m}^{o b s}$ \\
\hline Side reactions & $<0.3 \% S_{m}^{o b s}$ \\
\hline \multicolumn{2}{|c|}{ In addition: no effect can mimic the signatu } \\
\hline
\end{tabular}

tributions to the modulated amplitude have been calculated as summarized in table 1; for detailed and quantitative discussions see refs. $[2,3,10]$.

In conclusion, the presence of an annual modulation in the residual rate of the single-hit events in the lowestenergy interval (2-6) $\mathrm{keV}$, satisfying all the features expected for a Dark Matter particle component in the galactic halo is supported by the data of the seven annual cycles at $6.3 \sigma$ C.L. No systematic effect or side reaction able to account for the observed effect has been found. This is the experimental result of DAMA/NaI; it is modelindependent. No other experiment, whose result can be directly compared with this one in a model-independent way, is available so far in the field of Dark Matter investigation.

\section{Some corollary model-dependent quests for a candidate}

On the basis of the obtained $6.3 \sigma$ model-independent result, corollary investigations can also be pursued on the nature of the Dark Matter particle candidate. This latter investigation is instead model-dependent and - considering the large uncertainties which exist on the astrophysical, nuclear and particle physics assumptions and on the parameters needed in the calculations - has no general meaning (as is also the case of exclusion plots and of the Dark Matter particle parameters evaluated in indirect detection experiments). Thus, it should be handled in the most general way as we have pointed out with time passing $[6,7,8,9,10,11,12,13,2,3]$.

Candidates, kinds of Dark Matter particle couplings with ordinary matter and implications, cross-sections, nuclear form factors, spin factors, scaling laws, halo models, etc., are discussed to some extent in refs. [2,3]. The reader can find in this latter paper and in references therein devoted discussions to correctly understand the results ob-

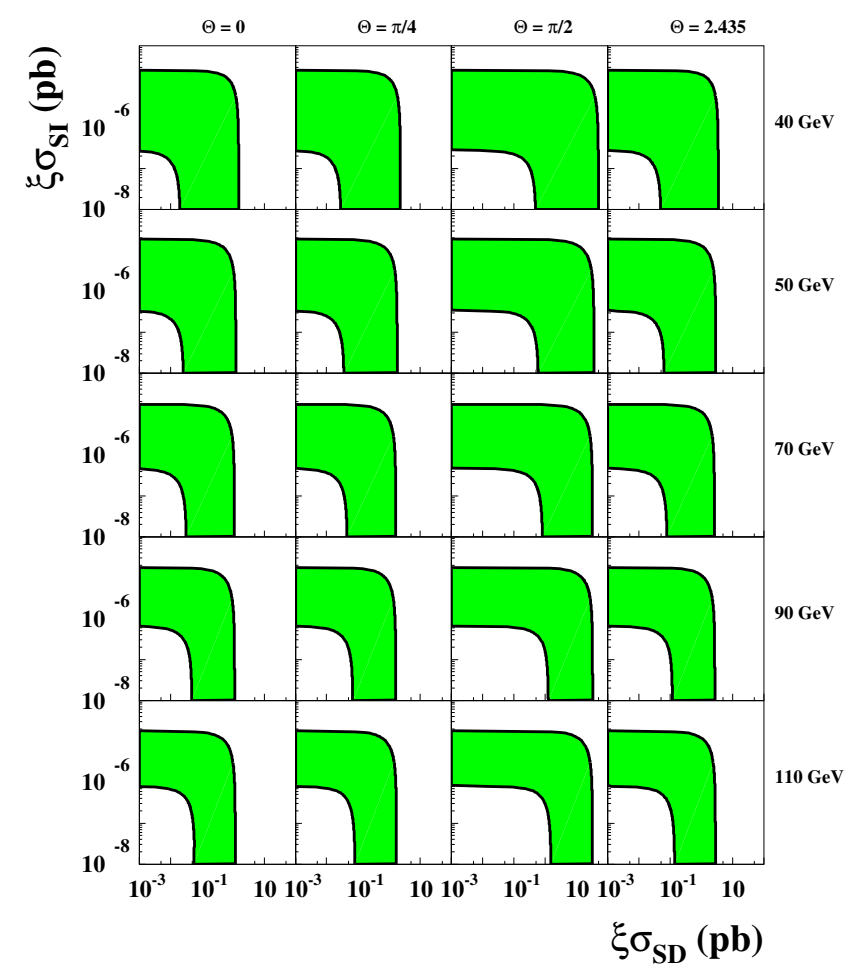

Fig. 2. Case of a Dark Matter particle with mixed SpinIndependent and Spin-Dependent interaction for the model frameworks given in refs. [2,3]. Coloured areas: example of slices (of the 4-dimensional allowed volume) in the plane $\xi \sigma_{S I}$ $v s$. $\xi \sigma_{S D}$ for some of the possible $m_{W}$ and $\theta$ values. Here, $\xi$ is the fractional amount of local density of Dark Matter particles, $\sigma_{S I}$ and $\sigma_{S D}$ are the point-like Spin-Independent and Spin-Dependent DM particle-nucleon cross-sections and $\theta$, defined in the $[0, \pi)$ interval, is an angle whose tangent is the ratio between the effective DM particle-nucleon coupling strengths for the Spin-Dependent interaction.

tained in corollary quests and the real validity of any claimed model-dependent comparison in the field. Here, we just remind that the results briefly summarized here are not exhaustive of the many scenarios possible at the present level of knowledge, including those depicted in some more recent works such as, e.g., refs. [19,20].

DAMA/NaI is intrinsically sensitive both to low and high Dark Matter particle mass having both a light (the ${ }^{23} \mathrm{Na}$ ) and a heavy (the ${ }^{127} \mathrm{I}$ ) target nucleus; in previous corollary quests for the candidate, Dark Matter particle masses above $30 \mathrm{GeV}$ (25 GeV in ref. [6]) have been presented $[7,9,11,12,13]$ for some model frameworks. Here, the present model-dependent lower bound quoted by LEP for the neutralino in the supersymmetric schemes based on GUT assumptions (37 GeV [21]) is just marked in fig. 3 . This model-dependent LEP limit — when considered - selects the Dark Matter particle-iodine elastic scatterings as dominant. In any case also other scenarios have been considered $^{2}$.

\footnotetext{
${ }^{2}$ In fact, when the assumption on the gaugino mass unification at GUT scale is released, neutralino masses down to $\simeq 6 \mathrm{GeV}$ are allowed $[22,23$.
} 

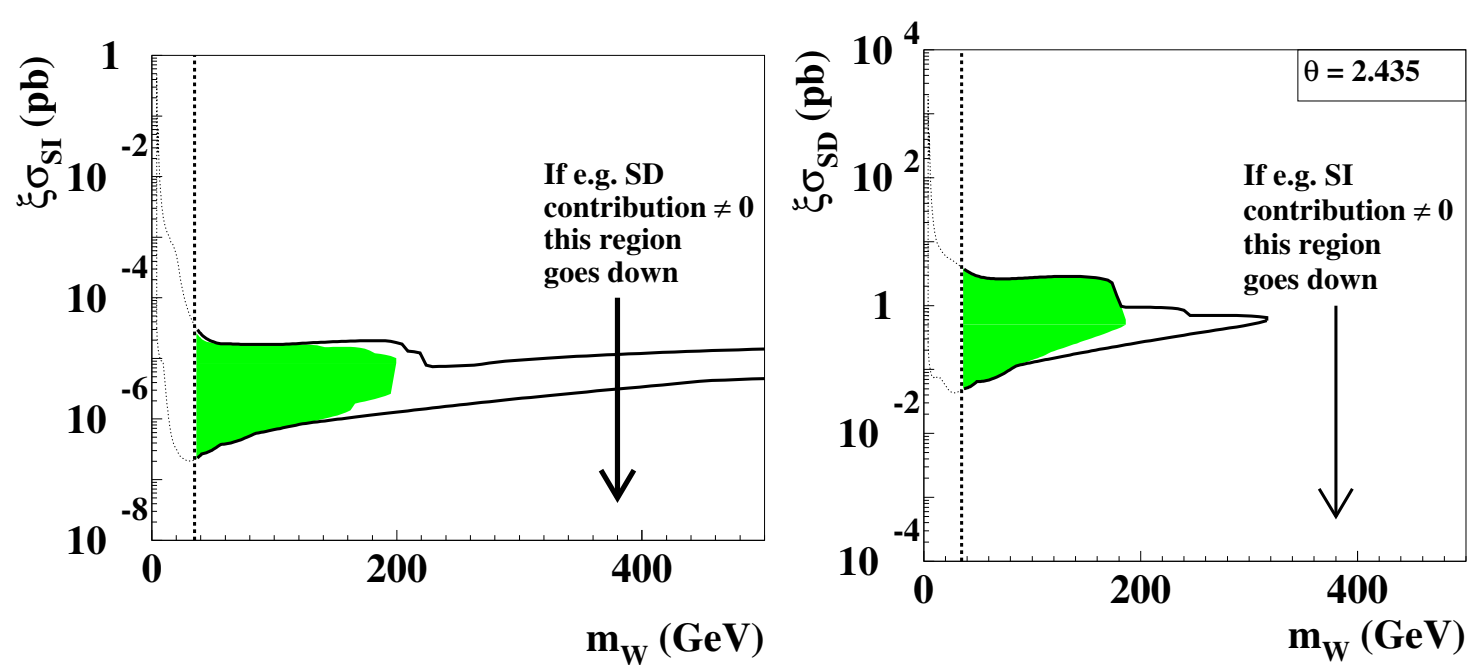

Fig. 3. On the left: case of a Dark Matter particle with dominant Spin-Independent interaction for the model frameworks given in refs. [2,3]. Region allowed in the plane $\left(m_{W}, \xi \sigma_{S I}\right)$. The vertical dotted line represents a bound in case of a neutralino candidate when supersymmetric schemes based on GUT assumptions are adopted to analyse the LEP data; the low mass region is allowed for the neutralino when other schemes are considered (see text) and for every other Dark Matter particle candidate. While the area at Dark Matter particle masses above $200 \mathrm{GeV}$ is allowed only for few configurations, the lower one is allowed by most configurations (the colored region gathers only those above the vertical line). Values of $\xi \sigma_{S I}$ lower than those corresponding to this allowed region are possible also, e.g., in case of an even small Spin-Dependent contribution. On the right: case of a Dark Matter particle with dominant Spin-Dependent interaction in the model frameworks given in refs. [2,3]. Example of a slice (of the 3 -dimensional allowed volume) in the plane $\left(m_{W}, \xi \sigma_{S D}\right)$ at a given $\theta$ value $(\theta$ is defined in the $[0, \pi)$ range); here $\theta=2.435$ ( $Z_{0}$ coupling). Values of $\xi \sigma_{S D}$ lower than those corresponding to this allowed region are possible also, e.g., in case of an even smaller Spin-Independent contribution.

For simplicity, here the results of these corollary quests for a candidate particle are presented in terms of allowed volumes/regions obtained as superposition of the configurations corresponding to likelihood function values distant more than $4 \sigma$ from the null hypothesis (absence of modulation) in each of the possible model frameworks considered in refs. [2,3]. These allowed regions take into account the time and energy behaviours of the single-hit experimental data and have been obtained by a maximumlikelihood procedure which requires the agreement i) of the expectations for the modulated part of the signal with the measured modulated behaviour for each detector and for each energy bin; ii) of the expectations for the unmodulated component of the signal with the respect to the measured differential energy distribution and - since ref. [9] - also with the bound on recoils obtained by pulse shape discrimination from the devoted DAMA/NaI-0 data [4]. The latter one acts in the likelihood procedure as an experimental upper bound on the unmodulated component of the signal and - as a matter of fact - as an experimental lower bound on the estimate of the background levels. Thus, the C.L.'s, which we quote for the allowed volumes/regions, already account for compatibility with the measured differential energy spectrum and with the measured upper bound on recoils. Finally, it is worth noting that the best-fit values of cross-sections and Dark Matter particle mass span over a large range when varying the considered model framework.

Figures 2-4 show some of the obtained allowed slices/regions $[2,3]$. Here we only recall that $\operatorname{tg} \theta$ is the ratio

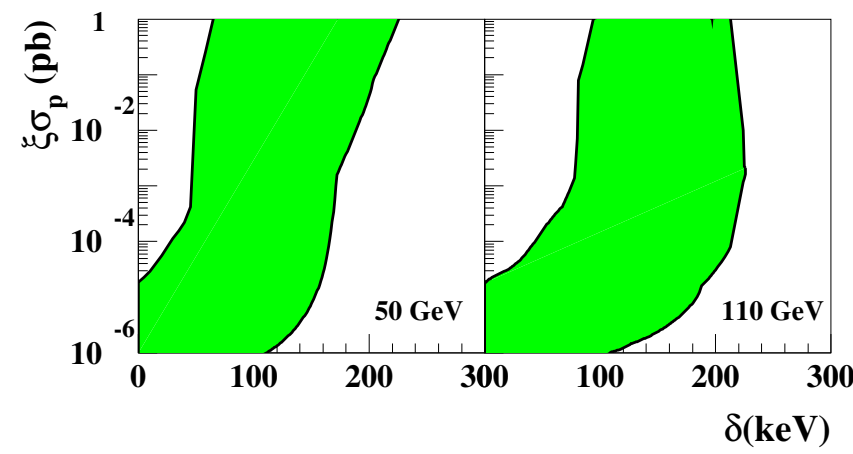

Fig. 4. Case of a Dark Matter particle with preferred inelastic interaction in the model frameworks given in refs. [2,3]. Examples of slices (coloured areas) of the 3-dimensional allowed volume $\left(\xi \sigma_{p}, \delta, m_{W}\right)$ for some $m_{W}$ values. Here, $\delta$ is the mass splitting of the Dark Matter candidate.

between the Dark Matter particle-neutron and the Dark Matter particle-proton effective Spin-Dependent coupling strengths and that $\theta$ is defined in the $[0, \pi)$ interval. Obviously, larger sensitivities than those reported in these figures would be reached when including the effect of other existing uncertainties on the astrophysical, nuclear and particle physics assumptions and related parameters; similarly, the set of the best-fit values would also be enlarged as well.

In fig. 5 the theoretical expectations in the purely SpinIndependent coupling for the particular case of a neu- 


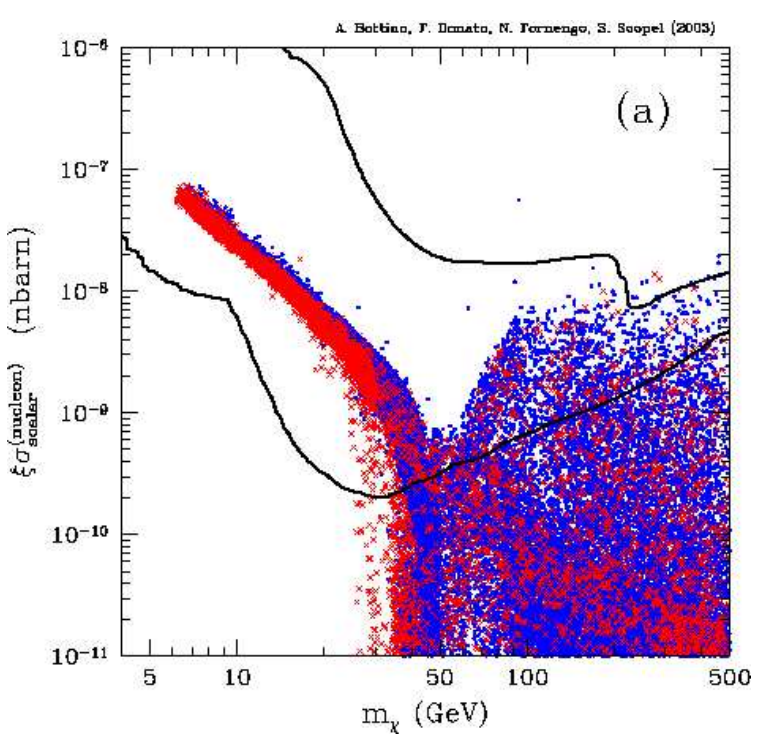

Fig. 5. Figure taken from ref. [23]: theoretical expectations of $\xi \sigma_{S I}$ vs. $m_{W}$ in the purely SI coupling for the particular case of a neutralino candidate in MSSM with gaugino mass unification at GUT scale released; the curve is the same as in fig. 3, left.

tralino candidate in MSSM with gaugino mass unification at GUT scale released [23] are shown. The marked curve surrounds the DAMA/NaI purely SI allowed region as in fig. 3, left.

On the other hand, some positive hints are present in indirect detection experiments; in fact, an excess of positrons and of gammas in the space has been reported with the respect to a modelled background; they are not in contradiction with the DAMA/NaI result. Moreover, recently, it has been suggested [24] that these positive hints and the effect observed by DAMA/NaI can also be described in a scenario with multi-component Dark Matter in the galactic halo, made of a subdominant component of heavy neutrinos of the 4 th family and of a sterile dominant component [24].

\section{Towards the future: from DAMA/Nal to DAMA/LIBRA and beyond}

The large merits of highly radiopure $\mathrm{NaI}(\mathrm{Tl})$ setup have been demonstrated in the practice by DAMA/NaI which has been the most radiopure setup available in this particular field. It has effectively pursued a model-independent approach to investigate Dark Matter particles in the galactic halo collecting an exposure several orders of magnitude larger than those available in the field and has obtained many other complementary or by-products results.

In 1996 DAMA proposed to realize a ton setup [25] and a new $R \& D$ project for highly radiopure $\mathrm{NaI}(\mathrm{Tl})$ detectors was funded at that time and carried out for several years in order to realize as an intermediate step the secondgeneration experiment, successor of DAMA/NaI, with an exposed mass of about $250 \mathrm{~kg}$.

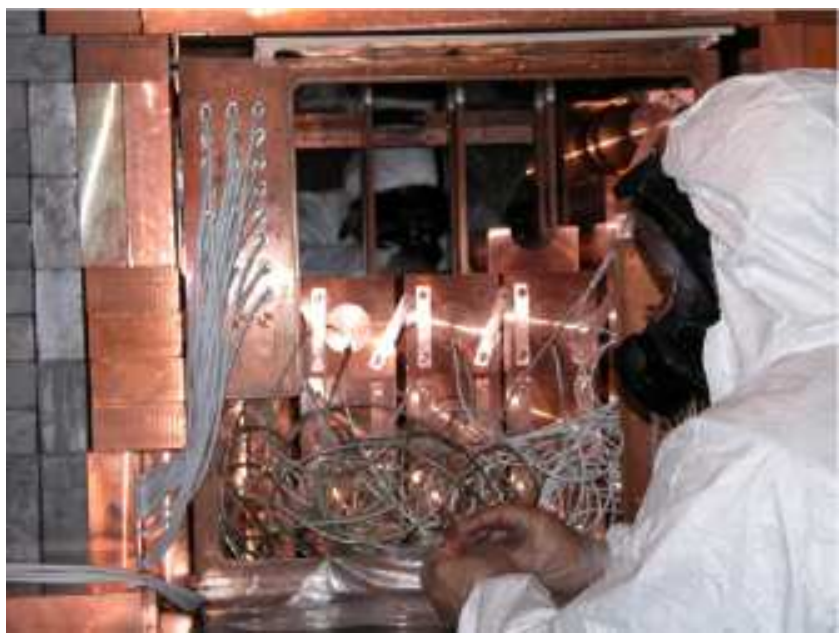

Fig. 6. The installation of the $25 \mathrm{NaI}(\mathrm{Tl})$ crystals $(9.70 \mathrm{~kg}$ each) of DAMA/LIBRA in high-purity nitrogen atmosphere. All the procedures as well as the photos have been carried out in high-purity nitrogen atmosphere.

Thus, new powders and other materials have been selected, new chemical/physical radiopurification procedures in NaI and TlI powders have been exploited, new growing/handling protocols have been developed and new prototypes have been built and tested. As a consequence of the results of this second-generation $\mathrm{R} \& \mathrm{D}$, the new experimental setup DAMA/LIBRA (Large sodium Iodide Bulk for RAre processes), $\simeq 250 \mathrm{~kg}$ highly radiopure $\mathrm{NaI}(\mathrm{Tl})$ crystal scintillators (matrix of twenty-five $\simeq 9.70 \mathrm{~kg}$ $\mathrm{NaI}(\mathrm{Tl})$ crystals), was funded at the end of 1999 and realised. In fact, after the completion of the DAMA/NaI data taking in July 2002, the dismounting of DAMA/NaI occurred and the installation of DAMA/LIBRA started. The experimental site as well as many components of the installation itself have been implemented (environment, shield of the photomultipliers, wiring, high-purity nitrogen system, cooling water of air conditioner, electronics and data acquisition system, etc.). In particular, all the copper parts have been chemically etched before their installation following a new devoted protocol and maintained in high-purity nitrogen atmosphere until the installation. All the procedures performed during the dismounting of DAMA/NaI and the installation of DAMA/LIBRA detectors have been carried out in high-purity nitrogen atmosphere (see fig. 6).

DAMA/LIBRA has taken data since March 2003 and the first data release will, most probably, occur when an exposure larger than that of DAMA/NaI will have been collected and analysed in all the aspects. The highly radiopure DAMA/LIBRA setup is a powerful tool for further investigation on the Dark Matter particle component in the galactic halo having many intrinsic merits $[1,2,3$, $4,5,6,7,8,9,10,11,12,13,14]$ and a larger exposed mass, an higher overall radiopurity and improved performances with the respect to DAMA/NaI. Thus, DAMA/LIBRA will further investigate the $6.3 \sigma$ C.L. model-independent evidence pointed out by DAMA/NaI with increased sensitivity in order to reach even higher C.L. Moreover, it 
will also offer an increased sensitivity to improve corollary quests on the nature of the candidate particle, trying to disentangle at least some of the many different possible astrophysical, nuclear and particle physics models as well as to investigate other new possible scenarios. As an example, we remind the effects induced on the Dark Matter particles distribution by the contributions from satellite galaxies tidal streams, by the possible existence of caustics and by the possible existence of "solar wakes" [26]. In particular, recently it has been pointed out [19] that contributions to the Dark Matter particles in the galactic halo should be expected from tidal streams from the Sagittarius Dwarf elliptical galaxy. Considering that this galaxy was undiscovered until 1994 and considering galaxy formation theories, one has to expect that also other satellite galaxies do exist and contribute as well. In particular, the Canis Major satellite Galaxy has been pointed out as reported in 2003 in ref. [27]; it can, in principle, play a very significant role being close to our galactic plane. At present, the best way to investigate the presence of a stream contribution is to determine more accurately the phase of the annual modulation, $t_{0}$, as a function of the energy; in fact, for a given halo model, $t_{0}$ would be expected to be (slightly) different from $\simeq 152.5 \mathrm{~d}$ and to vary with energy.

Moreover, other interesting topics will be addressed by the highly radiopure DAMA/LIBRA, such as the study i) on the velocity and spatial distribution of the Dark Matter particles in the galactic halo $[2,13]$; ii) on possible structures as clumpiness with small scale size; iii) on the couplings of the Dark Matter particle with the ${ }^{23} \mathrm{Na}$ and ${ }^{127}$ I target nuclei; iv) on the nature of the Dark Matter particles; v) on scaling laws and cross-sections (recently, it has been pointed out [20] that, even for the neutralino candidate, the usually adopted scaling laws could not hold); etc. A large work will be faced by DAMA/LIBRA, which will also further investigate with higher sensitivity several other rare processes.

Finally, at present a third-generation R\&D effort towards a possible $\mathrm{NaI}(\mathrm{Tl})$ ton setup has been funded and related works have already been started.

\section{Conclusion}

DAMA/NaI has been a pioneer experiment investigating as first the Dark Matter particle annual modulation signature with suitable sensitivity and control of the running parameters. During seven independent experiments of one year each, it has pointed out at $6.3 \sigma$ C.L. in a model-independent way the presence of a modulation satisfying the many peculiarities of an effect induced by Dark Matter particles in the galactic halo; no systematic effect or side reaction able to account for the observed effect has been found. As a corollary result, it has also pointed out the complexity of the quest for a candidate particle mainly because of the present poor knowledge on the many astrophysical, nuclear and particle physics related aspects. At present after a devoted R\&D effort, the second-generation DAMA/LIBRA ( $\simeq 250 \mathrm{~kg}$ more radiopure $\mathrm{NaI}(\mathrm{Tl})$ setup) has been realised and put in op- eration since March 2003 and a third-generation R\&D toward a possible ton $\mathrm{NaI}(\mathrm{Tl})$ setup, which we proposed in 1996 [25], is also in progress.

\section{References}

1. R. Bernabei et al., Nuovo Cimento A 112, 545 (1999).

2. R. Bernabei et al., Riv. Nuovo Cimento 26, 1 (2003).

3. R. Bernabei et al., Int. J. Mod. Phys. D 13, 2127 (2004).

4. R. Bernabei et al., Phys. Lett. B 389, 757 (1996).

5. R. Bernabei et al., Nuovo Cimento A 112, 1541 (1999).

6. R. Bernabei et al., Phys. Lett. B 424, 195 (1998).

7. R. Bernabei et al., Phys. Lett. B 450, 448 (1999).

8. P. Belli et al., Phys. Rev. D 61, 023512 (2000).

9. R. Bernabei et al., Phys. Lett. B 480, 23 (2000).

10. R. Bernabei et al., Eur. Phys. J. C 18, 283 (2000).

11. R. Bernabei et al., Phys. Lett. B 509, 197 (2001).

12. R. Bernabei et al., Eur. Phys. J. C 23, 61 (2002).

13. P. Belli et al., Phys. Rev. D 66, 043503 (2002).

14. R. Bernabei et al., Phys. Lett. B 408, 439 (1997); P. Belli et al., Phys. Lett. B 460, 236 (1999); R. Bernabei et al., Phys. Rev. Lett. 83, 4918 (1999); P. Belli et al., Phys. Rev. C 60, 065501 (1999); R. Bernabei et al., Phys. Lett. B 515, 6 (2001); F. Cappella et al., EPJdirect C 14, 1 (2002); R. Bernabei et al., Eur. Phys. J. A 23, 7 (2005); R. Bernabei et al., Eur. Phys. J. A 24, 51 (2005).

15. R. Bernabei et al., Nuovo Cimento A 103, 767 (1990); Nuovo Cimento C 19, 537 (1996); Astropart. Phys. 5, 217 (1996); Phys. Lett. B 387, 222 (1996); 389, 783(E) (1996); 436, 379 (1998); 465, 315 (1999); 493, 12 (2000); New J. Phys. 2, 15.1 (2000); Phys. Rev. D 61, 117301 (2000); EPJdirect C 11, 1 (2001); Nucl. Instrum. Methods A 482, 728 (2002); Phys. Lett. B 527, 182 (2002); 546, 23 (2002); in Beyond the Desert 03 (Springer, 2004) p. 541.

16. R. Bernabei et al., Astropart. Phys. 7, 73 (1997); R. Bernabei et al., Nuovo Cimento A 110, 189 (1997); P. Belli et al., Nucl. Phys. B 563, 97 (1999); P. Belli et al., Astropart. Phys. 10, 115 (1999); R. Bernabei et al., Nucl. Phys. A 705, 29 (2002); P. Belli et al., Nucl. Instrum. Methods A 498, 352 (2003); R. Cerulli et al., Nucl. Instrum. Methods A 525, 535 (2004).

17. K.A. Drukier et al., Phys. Rev. D 33, 3495 (1986); K. Freese et al., Phys. Rev. D 37, 3388 (1988).

18. D. Smith, N. Weiner, Phys. Rev. D 64, 043502 (2001).

19. K. Freese et al., Phys. Rev. Lett. 92, 11301 (2004).

20. G. Prezeau et al., Phys. Rev. Lett. 91, 231301 (2003).

21. K. Hagiwara et al., Phys. Rev. D 66, 010001 (2002).

22. A. Bottino et al., Phys. Rev. D 67, 063519 (2003); A. Bottino et al., hep-ph/0304080; D. Hooper, T. Plehn, MADPH-02-1308; CERN-TH/2002-29; hep-ph/0212226; G. Bélanger, F. Boudjema, A. Pukhov, S. Rosier-Lees, hepph/0212227.

23. A. Bottino et al., Phys. Rev. D 69, 037302 (2004).

24. K. Belotsky et al., hep-ph/0411093.

25. R. Bernabei et al., Astropart. Phys. 4, 45 (1995); R. Bernabei, Competitiveness of a very low radioactive ton scintillator for particle Dark Matter search, in The Identification of Dark Matter (World Scientific Publishers, 1997) p. 574.

26. F.S. Ling, P. Sikivie, S. Wick, astro-ph/0405231.

27. R.A. Ibata et al., Mon. Not. R. Astron. Soc. 348, 12 (2004). 